\title{
ASSEPSIA SUPERFICIAL DE SEMENTES DE CÁRTAMO COM USO DE HIPOCLORITO DE SÓDIO
}

\section{SURFACE ASEPSIS OF SHARPLE SEEDS WITH USE OF SODIUM HYPOCHLORITE}

\author{
Janine Farias Menegaes ${ }^{1}$ \\ Rogério Antônio Bellé ${ }^{2}$ \\ Ubirajara Russi Nunes ${ }^{3}$ \\ Geovana Facco Barbieri ${ }^{4}$
}

Submetido: 27/05/2020 / Aprovado: 27/10/2020 / Publicado: 31/10/2021.

\begin{abstract}
Resumo
O objetivo do presente trabalho foi avaliar tratamentos de assepsia e sua influência na qualidade fisiológica e sanitária de sementes de cártamo (Carthamus tinctorius L.). O experimento foi conduzido em delineamento inteiramente casualizado, em esquema fatorial 2x5 (soluções sanitizantes de hipoclorito de sódio $(\mathrm{NaClO})$ e períodos de imersão), com oito repetições. As soluções sanitizantes de $\mathrm{NaClO}$ foram constituídas de água destilada com $2 \%$ e $5 \%$ de cloro ativo, para um quilo de sementes. Os períodos de imersão das sementes nas soluções foram: $1 ; 5 ; 10 ; 15$ e 30 minutos. Avaliou-se a qualidade fisiológica das sementes através dos testes de germinação, emergência no campo, comprimento e massa de plântulas, sanidade, índices de velocidade de germinação e emergência. Constatou-se que o uso de $\mathrm{NaClO}$ demonstrou ser eficiente para a assepsia das sementes de cártamo, reduzindo a incidência de fitopatógenos e promovendo incremento na germinação.
\end{abstract}

Palavras-chave: Carthamus tinctorius L., Germinação, Soluções sanitizantes.

\begin{abstract}
The aim of the present study was to evaluate the asepsis procedures and their influence on the physiological and health quality of safflower seeds (Carthamus tinctorius L.). The experiment was carried out in a randomized isolated design, in a $2 \times 5$ factorial scheme (sodium hypochlorite sanitizing solutions $(\mathrm{NaClO})$ and immersion savings), with eight replications. The $\mathrm{NaClO}$ sanitizing solutions consisted of distilled water with $2 \%$ and $5 \%$ active chlorine, for one kilo of seeds. The seed immersion prices in the solutions were: $1 ; 5$; 10; 15 and 30 minutes. Eliminate the physiological quality of seeds through germination tests, field emergence, seedling length and mass, health, germination speed and emergence rates. It appears that the use of $\mathrm{NaClO}$ proved to be efficient for an asepsis of safflower seeds, captures of incidence of phytopathogens and promotion of increased germination.
\end{abstract}

Keywords: Carthamus tinctorius L., Germination, Sanitizing solutions.

\footnotetext{
${ }^{1}$ Doutora em Agronomia. Docente colaboradora do Departamento de Agronomia da UNICENTRO. E-mail: janine_rs@hotmail.com

2 Doutor em Fitotecnia. Docente aposentado do Departamento de Fitotecnia da UFSM. E-mail: rogeriobelle@gmail.com

${ }^{3}$ Doutor em Fitotecnia. Docente do Departamento de Fitotecnia da UFSM. E-mail: russinunes@yahoo.com.br

${ }^{4}$ Mestranda do Programa de Pós-Graduação em Fitossanidade pela UFP. E-mail: geovanafacco@hotmail.com DOI: http://dx.doi.org/10.24021/raac.v19i1.5523 


\section{INTRODUÇÃO}

O cártamo (Carthamus tinctorius L.) pertencente à família Asteraceae, sendo originário da Ásia, classifica-se como planta anual e rústica, com múltiplos propósitos de uso desde culinário, oleífero a ornamental. A cultura apresenta boa capacidade de adaptação a diferentes condições edafoclimáticas e baixa disponibilidade de água no solo. No Brasil, esta vem sendo utilizada como cultivo alternativo, especialmente para a produção de sementes, nos períodos de entressafra das culturas já consolidadas, como a da soja (Glycine max L.) (SANTOS; SILVA, 2015; YILMAZ et al., 2016).

As sementes de cártamo possuem teor de óleo em média de $35 \%$, rico em vitamina $\mathrm{C}, \mathrm{E}$, betacaroteno, ácido linoleico e oleico, amplamente utilizado nas indústrias alimentícias, farmacêuticas e petroleiras. Contudo, a baixa produtividade de sementes está associada à alta suscetibilidade ao ataque de patógenos, sobretudo, em ambientes de alta pluviosidade. As doenças folhares são os principais agentes depreciadores da qualidade ornamental na produção de hastes florais e, a incidência de patógenos associados a sementes é bastante expressiva, chegando a reduzir o rendimento de óleo para uso de biodiesel (MOURA et al., 2015; EMONGOR \& OAGILE, 2017).

A semente é um dos insumos agrícolas mais importante na implantação de uma lavoura, a suas qualidades fisiológica e sanitária podem interferirem desde o estabelecimento de plantas no campo até a produtividade final. Deste modo, sementes com alta incidência de fitopatógenos já advindas do campo, como a sementes de cártamo, necessitam de tratamento de assepsia superficial para garantir a expressão de sua qualidade genética (MARCOS FILHO, 2015; MENEGAES et al., 2019).

A utilização de métodos de assepsia em sementes tem como finalidade a diminuição ou eliminação superficial dos fitopatógenos, o qual se faz necessário para que se obtenham plântulas sadias, livre de fungos e bactérias e, posteriormente, plantas com capacidade de expressar seu máximo potencial produtivo. Entre os materiais mais utilizados para a assepsia, o hipoclorito de sódio, se configura como produto comum em laboratórios, devido fácil disponibilidade no mercado e ao baixo custo. Contudo, a exposição das sementes as soluções sanitizantes, deve ser realizado com uma concentração e um tempo específico para evitar a deterioração indevida das sementes, uma vez que esse produto apresenta alto potencial promotor de germinação e de superação de dormência (MEIRELES et al., 2007; PINHEIRO et al., 2016).

Neste contexto, o objetivo do presente trabalho foi avaliar tratamentos de assepsia superficial com hipoclorito de sódio e sua influência na qualidade fisiológica e sanitária de sementes de cártamo (Carthamus tinctorius L.).

\section{Material e Métodos}

\subsection{Delineamento experimental}

O experimento foi conduzido, no Laboratório Didático e de Pesquisas em Sementes, do Departamento de Fitotecnia no Campus da Universidade Federal de Santa Maria (UFSM), localizado em Santa Maria, RS (294' S; 534' W e altitude de $95 \mathrm{~m}$ ), em 2018. As sementes de cártamo foram cultivadas na safra 2017/2018 e colhidas em fevereiro de 2018. Após a colheita foram armazenadas em câmara fria $\left(15^{\circ} \mathrm{C}\right.$ e $40 \%$ UR) em sacos de papel Kraft (tipo pardo de $1 \mathrm{~kg}$ ), com grau de umidade médio de $9,0 \%$.

O delineamento adotado foi o inteiramente casualizado, em esquema fatorial $2 \times 5$ (soluções sanitizantes de hipoclorito de sódio $(\mathrm{NaClO})$ e período de imersão), com oito repetições, senda cada 
unidade experimental foi composta por 50 sementes. As soluções de $\mathrm{NaClO}$ foram constituídas de água destilada com 2 e $5 \%$ de cloro ativo, para um quilo de sementes. Os períodos de imersão das sementes nas soluções foram de um; cinco; $10 ; 15$ e 30 minutos.

Para a aplicação dos tratamentos, as sementes foram colocadas em frascos de vidro de 500 $\mathrm{mL}$ (tipo Becker) e adicionado as soluções sanitizantes de $\mathrm{NaClO}$ (com volume equivalente a 5\% da massa total das sementes) e permanecendo pelos períodos supracitados. Na sequência houve o enxague com água destilada para a remoção dos resíduos do produto.

\subsection{Análise da qualidade das sementes}

A caracterização do lote antes do tratamento (Tabela 1) e as avaliações qualitativas das sementes depois de tratadas foram pelos seguintes testes:

Teste padrão de germinação (TPG) e Índice de velocidade de germinação (IVG) foram realizados com oito repetições de 50 sementes, em rolo de papel de germinação, umedecido com água destilada na proporção de 2,5 vezes a massa do papel seco. Os rolos foram mantidos em germinador tipo Biochemical Oxygen Demand (BOD), com fotoperíodo de $24 \mathrm{~h}$ e temperatura de $25 \pm 2^{\circ} \mathrm{C}$. As avaliações foram realizadas aos 4 e aos 14 dias após a semeadura (DAS) (BRASIL, 2009a), e para o IVG utilizou-se como critério a germinação de plântulas normais apresentando alongamento da raiz primária e emergência dos cotilédones (ABUD et al., 2010).

Comprimento e massa seca de plântula: realizado com oito repetições de 20 sementes, as sementes que foram mantidas sob a mesma condição do TPG, aos 4 DAS foram medidos o comprimento da parte aérea e da radícula de dez plântulas normais de cada repetição, os resultados foram expressos em centímetros $(\mathrm{cm})$. Na sequência determinou-se massa seca total por secagem do material em estufa de ventilação forçada a $65 \pm 5^{\circ} \mathrm{C}$ por $48 \mathrm{~h}$, os resultados foram expressos em mg $\mathrm{pl}^{-1}$ (NAKAGAWA, 1999).

Emergência no campo e Índice de velocidade de emergência (IVE) foram realizados com oito repetições de 50 sementes que foram distribuídas em linhas de $1 \mathrm{~m}$, espaçadas a $0,2 \mathrm{~m}$ e com profundidade de $0,03 \mathrm{~m}$. A avaliação da emergência de plântulas foi realizada aos 14 DAS e para o IVE utilizou-se como critério a emergência de plântulas com desenvolvimento completo dos cotilédones e epicótilo (ABUD et al., 2010).

Para as variáveis de germinação e emergência das plântulas no campo, utilizou-se como referência a Instrução Normativa $\mathrm{n}^{\circ}$. 45/2013 do a cultura do girassol (Helianthus annuus L.), por pertencer à mesma família botânica do cártamo (Asteraceae), sendo exigidos valores 65-70\% (BRASIL, 2013).

Teste de sanidade foi realizado através da incubação em substrato de papel (Blotter Test), com oito repetições de 50 sementes. A semeadura ocorreu em caixas plásticas transparentes para germinação (gerbox), preparadas com três folhas de papel de germinação umedecido com água destilada correspondente a 2,5 vezes a massa do papel seco. A germinação foi inibida pelo método de congelamento por $24 \mathrm{~h}$. Posteriormente, as sementes permaneceram em BOD por cinco dias com fotoperíodo de $12 \mathrm{~h}$ de luz e $12 \mathrm{~h}$ de escuro, a temperatura de $20 \pm 2^{\circ} \mathrm{C}$. Foram avaliadas em lupa (microscópio estereoscópio) as percentagens de sementes infestadas e a identificação dos fitopatógenos em nível de gênero (BRASIL, 2009b).

Índice de incremento de germinação aos 14 DAS (II.GER): foi determinado pela metodologia de Menegaes et al. (2019), expressa na Equação 1

II.GER $=(($ GERts - GERc $) /$ GERc $) * 100$ 
em que: GERts: germinação do tratamento de sementes e GERc: germinação na caracterização do lote.

Índice de incremento de emergência de plântulas no campo (II.ECP): foi determinado pela metodologia de Menegaes et al. (2019), expressa na Equação 2

II.ECP $=(($ ECPts $-\mathrm{ECPc}) / \mathrm{ECPc}) * 100$

em que: ECPts: emergência do tratamento de sementes e ECPc: emergência na caracterização do lote.

Índice de controle de sementes infestadas totais (IC.SIT): foi determinado pela metodologia de Menegaes et al. (2019), expressa na Equação 3

IC.SIT $=(($ SITc - SITts $) /$ SITc $) * 100$

em que: SITts: sementes infestadas no tratamento de sementes e SITc: sementes infestadas na caracterização do lote.

\subsection{Análises estatísticas}

Os dados expressos em percentagem foram transformados em arco-seno $\sqrt{x / 100}$, e as análises de variância e a comparação das médias pelo teste Tukey $(\mathrm{p}<0,05)$, realizados com o auxílio do programa estatístico SISVAR (FERREIRA, 2014).

\section{RESUltados}

Verificou-se que a qualidade fisiológica inicial do lote de sementes de cártamo utilizado, neste trabalho, foi em média de $71 \%$ de germinação e $66 \%$ de emergência no campo (Tabela 1).

$\mathrm{Na}$ Tabela 2, observou-se que não houve interação significa para vários parâmetros fisiológicos relativos a germinação das sementes. Em relação a avaliação da germinação (14 DAS) e da emergência no campo realizada após o tratamentos de sementes, verificou-se que há a manutenção do padrão exigido pelo MAPA em todas as soluções sanitizantes de $\mathrm{NaClO}$ e nos períodos de imersão, promovendo um incremento no percentual germinativo e de emergência no campo.

Os índices de velocidade de germinação e emergência (IVG e IVE), comprimento e a massa seca (radicular e parte aérea) de plântulas não apresentaram, significância entre os fatores.

A qualidade sanitária das sementes na caracterização do lote (Tabela1) apresentou $61 \%$ de infestação, depois da realização dos tratamentos de sementes (Tabela3), observou-se uma redução da porcentagem de sementes totais infestadas, indicando uma melhoria na qualidade sanitária destas.

Os fitopatógenos de maior incidência identificados nas sementes foram os dos gêneros Aspergillus spp., Botrytis spp., Colletotrichum spp., Fusarium spp., Penicillium spp. e Sclerotinia spp. (Tabelas 1 e 3 ).

$\mathrm{Na}$ Tabela 4, verificou-se os tratamentos de assepsia das sementes apresentaram interação significativa quando houve a comparação do lote inicial de sementes (Tabela 1) e os índices de incremento de germinação aos 14 DAS (II.GER) e de emergência de plântulas no campo (II.ECP) e o índice de controle de sementes infestadas totais (IC.SIT). 


\section{DISCUSSÃo}

O lote de sementes de cártamo utilizado neste trabalho apresentou características de lote com potencial comercial segundo os padrões do MAPA (BRASIL, 2013), contudo, com grande incidência de fitopatógenos (61\%) (Tabela 1).

As soluções sanitizantes de $\mathrm{NaClO}$ utilizadas nos diferentes períodos de imersão, as quais as sementes foram submetidas não causam efeito fitotóxico as mesmas, tratando-as assepticamente (Tabela 2). Ruim et al. (2010) e Jesus et al. (2016) verificaram que o uso de $\mathrm{NaClO}$ em diferentes concentrações não causaram danos as sementes de café-conilon (Coffea canephora L.) e de mamão (Carica papaya L.), ao mesmo tempo que auxiliaram na remoção da camada de mucilagem que envolve estas sementes, beneficiando a expressão da sua germinação.

Teodoro el at. (2015) destacam que o uso de $1 \% \mathrm{NaClO}$ por 3 minutos foi o suficiente para propiciar o aumento da velocidade da germinação (IVG) em sete cultivares de sementes de alface (Lactuca sativa L.), sem efeitos tóxicos as mesmas.

Marcos Filho (2015) relata que testes de vigor como IVG, IVE, comprimento e massa seca de plântulas são parâmetros biométricos para avaliação dos possíveis efeitos tóxicos dos produtos utilizados no tratamento de sementes, sendo essenciais para a mensuração do estabelecimento do estande de plântulas no campo. Pinheiro et al. (2016) verificaram que nenhum dos tratamentos de assepsia testados influenciou o desenvolvimento das plântulas das espécies acácia (Senegalia bonariensis Seigler \& Ebinger.), angico (Parapiptadenia rigida (Benth.) Brenan.), cedro (Cedrela fissilis Vell.) e pata-de-vaca (Bauhinia forficata Link.).

Verificou-se que houve uma melhoria do percentual da germinação das sementes de cártamo, sugerindo que a imersão nessas soluções beneficia a assepsia destas sementes, como observado pelos percentuais de sementes infestadas totais na caracterização do lotes era de $61 \%$ passando para uma média de $12 \%$ com $5 \% \mathrm{NaClO}$ por 30 min de imersão na solução. Assim, a baixa incidência de fitopatógenos sobre as sementes de cártamo favoreceu a expressão do potencial germinativo das mesmas (Tabelas 2 e 3 ).

A qualidade sanitária das sementes de cártamo foi beneficiada com a imersão destas em soluções de $\mathrm{NaClO}$, com redução das sementes totais infestadas (Tabela 3). Muniz et al. (2007) também verificaram redução acentuada de fungos nas sementes de espécies florestais de angico (Parapiptadenea rigida (Benth.) Brenan), canafístula (Pelptophorum dubium (Spreng.) Taub.), maricá (Mimosa bimucronata (DC.) O. Kuntze), pau-fava (Cassia multijuga (Rich) H.S. Irwing e Barneby) e timbaúva (Entereolobium contortisiliquum (Vell.) Morong), com a utilização de $\mathrm{NaClO}$ como método de assepsia.

Oliveira et al. (2012) verificaram que o $\mathrm{NaClO}$ tem potencial no tratamento de sementes visando à remoção de fitopatógenos na superficial destes. Pois, a associação de microrganismos às sementes via tecidos externos se configura como uma das principais formas de desinfestação. Ainda corroborando com os resultados deste trabalho, Souza et al. (2011) observaram além de maiores porcentagens de germinação de sementes de guabijú (Myrcianthes pungens Berg.), boa desinfecção destas quando submetidas a soluções com concentrações de 4 e $6 \%$ de $\mathrm{NaClO}$. Pinheiro et al. (2016) verificaram que $2 \% \mathrm{NaClO}$ por um minuto proporcionou assepsia para diferentes sementes de espécies florestais (acácia, angico, cedro e pata-de-vaca), sem danos na germinação e com a redução da incidência de Fusarium spp. e Penicillium spp. sobre essas sementes.

Souza et al. (2013) verificaram que o uso de $2 \% \mathrm{NaClO}$ pelos períodos de 15 minutos é o suficiente para eliminar fungos presentes em sementes de copaíba (Copaifera langsdorffii Desf.). Medeiros et al. (2015) verificaram que eficiência do controle asséptico sobre sementes resulta na 
melhoria do seu potencial germinativo, tanto em condições de laboratório como de campo, corroborando com os resultados deste trabalho.

A comparação dos índices de II.GER, II.ECP e IC.SIT (Tabela 4) com o lote inicial de sementes de cártamo (Tabela 1), verificou-se que interação positiva tanto para a expressão do potencial de germinação das sementes como a sua assepsia. Entre os tratamentos, destacam-se as soluções sanitizantes com $5 \%$ de $\mathrm{NaClO}$ em todos os períodos, por apresentarem melhores resultados.

Medeiros et al. (2015) e Menegaes et al. (2019) observaram que conforme a eficiência do controle fúngico sobre as superfície das sementes há uma redução da sua microflora, afetando positivamente a sua qualidade fisiológica, com o aumento do percentual germinativo tanto em condições de laboratório como no campo, corroborando com os resultados deste trabalho. Oliveira et al. (2012) e Pinheiro et al. (2016) verificaram que a eficiência do uso de $\mathrm{NaClO}$ para tratamentos de assepsia de sementes, além de reduzir a incidência de fitopatógenos, também favorece a germinação e o estabelecimento das plântulas no campo.

Portanto, o tratamento de sementes de cártamo com uso de hipoclorito de sódio $(\mathrm{NaClO})$ demonstrou ser eficiente para a assepsia das sementes, com redução da incidência de fitopatógenos e proporcionando um incremento na germinação, quando comparado a ausência de sanitização.

\section{AgRadecimentos}

A CAPES (Coordenação de Aperfeiçoamento de Pessoal de Nível Superior) pelo incentivo e financiamento deste trabalho e, ao Programa de Pós-Graduação em Agronomia da Universidade Federal de Santa Maria. Ao CNPq (Conselho Nacional de Desenvolvimento Científico e Tecnológico) pela bolsa de produtividade ao professor orientador deste trabalho.

\section{REFERÊNCIAS}

ABUD HF, GONÇALVES NG, REIS RGES, GALLÃO MI, INNECCO R. Morfologia de sementes e plântulas de cártamos. Revista Ciência Agronômica, Fortaleza, v. 41, n. 2, p. 259-265, 2010.

BRASIL. Ministério da Agricultura, Pecuária e Abastecimento. Instrução Normativa n. 45. Brasília. MAPA. 2013, 38 p.

BRASIL. Ministério da Agricultura, Pecuária e Abastecimento. Manual de Análise Sanitária de Sementes. Brasília: MAPA, 2009b. 200 p.

BRASIL. Ministério da Agricultura, Pecuária e Abastecimento. Regras para Análise de Sementes. Brasília: MAPA, 2009a. 395p.

EMONGOR V, OAGILE O. Safflower production. Botswana: The Regional Universities Forum for Capacity Building in Agriculture - RUFORUM. 2017. 6 7p.

FERREIRA DF. Sisvar: A guide for its bootstrap procedures in multiple comparisons. Ciência e Agrotecnologia, Lavras, v. 38, n. 2, p. 109-112, 2014. 
JESUS VAM, ARAÚJO EF, NEVES AA, SANTOS FL, DIAS LAS, SILVA RF. Ratio of seeds and sodium hypochlorite solution on the germination process of papaya seeds. Journal of Seed Science, Londrina, v. 38, n. 1, p. 057-061, 2016.

MARCOS FILHO J. Fisiologia de sementes de plantas cultivadas. ABRATES: Londrina, 2015. $650 \mathrm{p}$.

MEDEIROS JGF, NETO ACA, SILVA EC, HUANG MN, NASCIMENTO LC. Qualidade sanitária de sementes de Caesalpinia ferrea: incidência de fungos, controle e efeitos na qualidade fisiológica com o uso de extratos vegetais. Floresta, Curitiba, v.45, n.1, p.163-174, 2015.

MEIRELES RC, ARAÚJO EF, REIS MS, SEDIYAMA CS, SAKIYAMA NS, REIS LS. Secafé: Metodologia para acelerar a germinação das sementes de café. Revista Brasileira de Sementes, Londrina, v. 29, n. 3, p. 80-86, 2007.

MENEGAES JF, NUNES UR, BERTAGNOLLI CM, MUNIZ MFB, BELLÉ RA, BACKES FAAL. Physiological and sanitary quality of safflower seeds under differentseed treatments. Journal of Agricultural Studies, Las Vegas, v. 7, n. 4, p.2 82-296, 2019.

MOURA PCS, BORTOLHEIRO FPAP, GUIMARÃES TM, LEAL DPV, SILVA MA. Características gerais e ecofisiologia do cártamo (Carthamus tinctorius). Journal of Agronomic Sciences, Umuarama, v. 4, n. esp., p. 136-150, 2015.

MUNIZ MFB, SILVA LM, BLUME E. Influência da assepsia e do substrato na qualidade de sementes e mudas de espécies florestais. Revista Brasileira de Sementes, Londrina, v. 29, n. 1, p. 140-146, 2007.

NAKAGAWA J. Testes de vigor baseados na avaliação das plântulas. In: KRZYZANOSWKI, F.C.; VIEIRA, R.D.; FRANÇA NETO. J.B. Vigor de sementes: conceitos e testes. Londrina: ABRATES. 1999. $218 \mathrm{p}$.

OLIVEIRA JD, SILVA JB, DAPONT EC, SOUZA LMS, RIBEIRO SA. Métodos para detecção de fungos e assepsia de sementes de Schizolobium amazonicum (Caesalpinioideae). Bioscience Journal, Uberlândia, v. 28, n. 6, p. 945-953, 2012

PINHEIRO CG, LAZAROTTO M, MUNIZ MFB, REDIN CG, SANTOS MV. Efeito da assepsia superficial na germinação e incidência de fungos em sementes de espécies florestais. Pesquisa Florestal Brasileira, Colombo, v. 36, n. 87, p. 253-260, 2016.

RUBIM RF, VIEIRA HD, ARAÚJO EF, VIANA AP, COELHO FC. Tratamento com hipoclorito de sódio para remoção do pergaminho e aceleração da germinação de sementes de café conilon. Revista Brasileira de Sementes, Londrina, v. 32, n. 4, p. 088-098, 2010.

SANTOS RF, SILVA MA. Carthamus tinctorius L.: Uma alternativa de cultivo para o Brasil. Acta Iguazu, Cascavel, v. 4, n. 1, p. 26-35, 2015.

SOUZA LMS, SILVA JB, GOMES NSB. Qualidade sanitária e germinação de sementes de copaíba. Bioscience Journal, Uberlândia, v. 29, n. 1, p. 1524-1531, 2013. 
SOUZA LS, FIOR CS, SOUZA PVD, SCHWARZ SF. Desinfeção de sementes e multiplicação de in vitro de guabijuzeiro a partir de segmentos apicais juvenis (Myrcianthes pungens O. Berg). Revista Brasileira de Fruticultura, Jaboticabal, v. 33, n. 3, p. 691-697, 2011.

TEODORO MS, ALVES MCS, SEIXAS FJS, LACERDA MN, ARAÚJO LMS. Influência do $\mathrm{NaClO}$ na germinação de sementes de alface em Parnaíba-PI. Revista Verde de Agroecologia e Desenvolvimento Sustentável, Pombal, v. 10, n. 4, p. 33-37, 2015.

YILMAZ H, COMAK MB, TURGUT F. Analysis of factors related to farmers' benefiting from safflower (Carthamus Tinctorius L., ) production support: the case of central anatolia in Turkey. The Journal of Animal \& Plant Sciences, Islamabade, v. 26, n. 5, p. 1411-1417, 2016. 
Tabela 1. Caracterização do lote de sementes de cártamo.

\begin{tabular}{cc}
\hline Características do lote & \\
\hline Germinação aos 4 DAS (\%) & 38 \\
Germinação 14 DAS (\%) & 71 \\
Emergência no campo (\%) & 66 \\
Índice de velocidade de germinação (IVG) & 43,22 \\
Índice de velocidade de emergência (IVE) $^{-1}$ & 23,228 \\
Massa seca radicular de plântula (mg pl $\left.^{-1}\right)$ & 1,99 \\
Comprimento radicular de plântula (cm) & 5,9 \\
Comprimento da parte aérea de plântula (cm) & 2,5 \\
Massa seca da parte aérea de plântula (mg pl $\left.{ }^{-1}\right)$ & 10 \\
Sementes infestadas totais (\%) & 61 \\
Aspergillus spp. (\%) & 25 \\
Botrytis spp. (\%) & 24 \\
Colletotrichum spp. (\%) & 15 \\
Fusarium spp. (\%) & 16 \\
Penicillium spp. (\%) & 9 \\
Sclerotinia spp. (\%) & 11
\end{tabular}

Tabela 2. Assepsia superficial de sementes de cártamo submetidas a diferentes soluções sanitizantes de hipoclorito de sódio $(\mathrm{NaClO})$ e períodos de imersão.

\begin{tabular}{|c|c|c|c|c|c|c|}
\hline \multirow{2}{*}{$\mathrm{NaClO}$} & \multicolumn{6}{|c|}{ Períodos de imersão (min.) } \\
\hline & 1 & 5 & 10 & 15 & 30 & MD \\
\hline & \multicolumn{6}{|c|}{ Germinação aos 4 DAS (\%) } \\
\hline $2 \%$ & $42^{\text {ns }}$ & 43 & 43 & 46 & 40 & $43 \mathrm{~A}$ \\
\hline $5 \%$ & 43 & 43 & 45 & 45 & 40 & $43 \mathrm{~A}$ \\
\hline MD & $43 \mathrm{c}$ & $43 \mathrm{c}$ & $44 \mathrm{~b}$ & $46 a$ & $40 \mathrm{~d}$ & \\
\hline \multirow[t]{2}{*}{$\mathrm{CV}(\%)$} & \multicolumn{6}{|c|}{6,18} \\
\hline & \multicolumn{6}{|c|}{ Germinação aos 14 DAS (\%) } \\
\hline $2 \%$ & $76 \mathrm{Bc}^{*}$ & $77 \mathrm{Bc}$ & $80 \mathrm{Bb}$ & $85 \mathrm{Aa}$ & $75 \mathrm{Ac}$ & 79 \\
\hline $5 \%$ & $78 \mathrm{Ab}$ & $83 \mathrm{Aa}$ & $85 \mathrm{Aa}$ & $85 \mathrm{Aa}$ & $76 \mathrm{Ab}$ & 81 \\
\hline MD & 78 & 80 & 83 & 85 & 76 & \\
\hline \multirow[t]{2}{*}{$\mathrm{CV}(\%)$} & \multicolumn{6}{|c|}{2,59} \\
\hline & \multicolumn{6}{|c|}{ Índice de velocidade de germinação (IVG) } \\
\hline $2 \%$ & $51,4^{\mathrm{ns}}$ & 56,4 & 53,4 & 54,2 & 50,4 & $53,6 \mathrm{~A}$ \\
\hline $5 \%$ & 45,6 & 54,4 & 56,4 & 50,4 & 45,6 & $50,5 \mathrm{~B}$ \\
\hline $\mathrm{MD}$ & $45,6 \mathrm{c}$ & $55,4 \mathrm{a}$ & $54,9 \mathrm{a}$ & $52,3 \mathrm{~b}$ & $48,0 \mathrm{c}$ & \\
\hline \multirow[t]{2}{*}{$\mathrm{CV}(\%)$} & \multicolumn{6}{|c|}{15,45} \\
\hline & \multicolumn{6}{|c|}{ Emergência no campo (\%) } \\
\hline $2 \%$ & $76 \mathrm{Bb}^{*}$ & $76 \mathrm{Bb}$ & $80 \mathrm{Ba}$ & $81 \mathrm{Aa}$ & $74 \mathrm{Bb}$ & 78 \\
\hline $5 \%$ & $80 \mathrm{Ab}$ & $79 \mathrm{Ab}$ & $82 \mathrm{Aa}$ & $76 \mathrm{Bc}$ & $76 \mathrm{Ac}$ & 79 \\
\hline MD & 80 & 78 & 81 & 79 & 75 & \\
\hline \multirow[t]{2}{*}{$\mathrm{CV}(\%)$} & \multicolumn{6}{|c|}{3,54} \\
\hline & \multicolumn{6}{|c|}{ Índice de velocidade de emergência (IVE) } \\
\hline $2 \%$ & $26,4^{\mathrm{ns}}$ & 26,4 & 26,2 & 26,1 & 26,8 & $26,4 \mathrm{~A}$ \\
\hline $5 \%$ & 26,4 & 25,2 & 25,7 & 26,2 & 26,5 & $26,0 \mathrm{~A}$ \\
\hline MD & $26,4 \mathrm{a}$ & $25,8 \mathrm{~b}$ & $26,0 \mathrm{~b}$ & $26,2 \mathrm{a}$ & $26,7 \mathrm{a}$ & \\
\hline \multirow[t]{2}{*}{$\mathrm{CV}(\%)$} & \multicolumn{6}{|c|}{8,22} \\
\hline & \multicolumn{6}{|c|}{ Massa seca da parte érea de plântula $\left(\mathrm{mg} \mathrm{pl}^{-1}\right)$} \\
\hline $2 \%$ & $12,9^{\mathrm{ns}}$ & 12,9 & 11,6 & 11,2 & 10,8 & $11,6 \mathrm{~A}$ \\
\hline $5 \%$ & 10,8 & 11,9 & 11,9 & 12,1 & 12,4 & $11,8 \mathrm{~A}$ \\
\hline MD & $10,8 \mathrm{c}$ & $12,4 \mathrm{a}$ & $11,8 \mathrm{~b}$ & $11,7 \mathrm{~b}$ & $11,6 \mathrm{~b}$ & \\
\hline \multirow[t]{2}{*}{$\mathrm{CV}(\%)$} & \multicolumn{6}{|c|}{17,35} \\
\hline & \multicolumn{6}{|c|}{ Massa seca radicular de plântula $\left(\mathrm{mg} \mathrm{pl}^{-1}\right)$} \\
\hline $2 \%$ & $2,25 \mathrm{~ns}$ & 2,3 & 2,3 & 2,3 & 2,5 & $2,3 \mathrm{~A}$ \\
\hline $5 \%$ & 2,3 & 2,4 & 2,2 & 2,5 & 2,5 & $2,4 \mathrm{~A}$ \\
\hline MD & $2,3 \mathrm{a}$ & $2,3 \mathrm{a}$ & $2,2 \mathrm{a}$ & $2,4 \mathrm{a}$ & $2,5 \mathrm{a}$ & \\
\hline \multirow[t]{2}{*}{$\mathrm{CV}(\%)$} & & & 11,7 & & & \\
\hline & & priment & radicul & de plâ & tula $(\mathrm{cm}$ & \\
\hline $2 \%$ & $3,2^{\mathrm{ns}}$ & 2,9 & 3,1 & 3,1 & 2,8 & $3,0 \mathrm{~A}$ \\
\hline $5 \%$ & 2,3 & 2,3 & 2,3 & 2,5 & 2,5 & $2,4 \mathrm{~B}$ \\
\hline MD & $2,3 \mathrm{~b}$ & $2,6 \mathrm{a}$ & $2,7 \mathrm{a}$ & $2,8 \mathrm{a}$ & $2,7 \mathrm{a}$ & \\
\hline CV(\%) & & & 10,5 & & & \\
\hline & Com & imento & parte & rea de & ântula & \\
\hline $2 \%$ & $6,5^{\mathrm{ns}}$ & 6,7 & 6,8 & 7,1 & 7,0 & $6,9 \mathrm{~B}$ \\
\hline $5 \%$ & 6,7 & 7,2 & 7,5 & 6,9 & 7,6 & $7,2 \mathrm{~A}$ \\
\hline MD & $6,7 \mathrm{c}$ & $7,0 \mathrm{~b}$ & $7,2 \mathrm{a}$ & $7,0 \mathrm{~b}$ & $7,3 \mathrm{a}$ & \\
\hline CV $(\%)$ & & & 10,1 & & & \\
\hline
\end{tabular}

*interação significativa e ${ }^{\text {ns }}$ interação não significativa dos fatores. Teste de médias não seguidas pela letra, maiúsculas na coluna e minúsculas na linha, diferem pelo teste de Tukey $(p<0,05)$. Coeficiente de variação (CV), Média (MD). 
Tabela 3. Incidência de fitopatógenos sobre as sementes de cártamo submetidas a diferentes soluções sanitizantes de hipoclorito de sódio $(\mathrm{NaClO})$ e períodos de imersão.

\begin{tabular}{|c|c|c|c|c|c|c|}
\hline \multirow{2}{*}{$\mathrm{NaClO}$} & \multicolumn{6}{|c|}{ Períodos de imersão (min.) } \\
\hline & 1 & 5 & 10 & 15 & 30 & MD \\
\hline & \multicolumn{6}{|c|}{ Sementes infestadas totais $(\%)$} \\
\hline $2 \%$ & $56 \mathrm{Aa}^{*}$ & $22 \mathrm{Ab}$ & $21 \mathrm{Ab}$ & $16 \mathrm{Ac}$ & $17 \mathrm{Ac}$ & 26 \\
\hline $5 \%$ & $52 \mathrm{Aa}$ & $20 \mathrm{Ab}$ & $22 \mathrm{Ab}$ & $18 \mathrm{Ab}$ & $12 \mathrm{Bc}$ & 25 \\
\hline MD & 56 & 30 & 26 & 22 & 20 & \\
\hline \multirow[t]{2}{*}{ CV $(\%)$} & \multicolumn{6}{|c|}{8,72} \\
\hline & \multicolumn{6}{|c|}{ Aspergillus spp. (\%) } \\
\hline $2 \%$ & $17 \mathrm{Bb}$ & $7 \mathrm{Bc}$ & $9 \mathrm{Bc}$ & $8 \mathrm{Bc}$ & $37 \mathrm{Aa}$ & 16 \\
\hline $5 \%$ & $21 \mathrm{Aa}$ & $16 \mathrm{Ab}$ & $17 \mathrm{Ab}$ & $16 \mathrm{Ab}$ & $5 \mathrm{Bc}$ & 15 \\
\hline MD & 21 & 12 & 13 & 13 & 21 & \\
\hline \multirow[t]{2}{*}{$\mathrm{CV}(\%)$} & \multicolumn{6}{|c|}{18,49} \\
\hline & \multicolumn{6}{|c|}{ Botrytis spp. (\%) } \\
\hline $2 \%$ & $19 \mathrm{Aa}$ & $15 \mathrm{Aa}$ & $16 \mathrm{Aa}$ & $16 \mathrm{Aa}$ & $15 \mathrm{Aa}$ & 16 \\
\hline $5 \%$ & $13 \mathrm{Bb}$ & $11 \mathrm{Ab}$ & $8 \mathrm{Bc}$ & $9 \mathrm{Bc}$ & $18 \mathrm{Aa}$ & 12 \\
\hline MD & 19 & 13 & 15 & 12 & 14 & \\
\hline \multirow[t]{2}{*}{$\mathrm{CV}(\%)$} & \multicolumn{6}{|c|}{19,75} \\
\hline & \multicolumn{6}{|c|}{ Colletotrichum spp. (\%) } \\
\hline $2 \%$ & $7 \mathrm{Bb}$ & $9 \mathrm{Ab}$ & $11 \mathrm{Ab}$ & $28 \mathrm{Aa}$ & $9 \mathrm{Ab}$ & 13 \\
\hline $5 \%$ & $11 \mathrm{Ab}$ & $6 \mathrm{Ac}$ & $16 \mathrm{Ab}$ & $21 \mathrm{Aa}$ & $5 \mathrm{Ac}$ & 12 \\
\hline $\mathrm{MD}$ & 11 & 9 & 16 & 26 & 6 & \\
\hline \multirow[t]{2}{*}{$\mathrm{CV}(\%)$} & \multicolumn{6}{|c|}{18,65} \\
\hline & \multicolumn{6}{|c|}{ Fusarium spp. (\%) } \\
\hline $2 \%$ & $25^{\mathrm{ns}}$ & 17 & 13 & 12 & 23 & $18 \mathrm{~B}$ \\
\hline $5 \%$ & 19 & 22 & 25 & 14 & 47 & $25 \mathrm{~A}$ \\
\hline $\mathrm{MD}$ & $20 \mathrm{~b}$ & $19 \mathrm{~b}$ & $22 \mathrm{~b}$ & $19 \mathrm{~b}$ & $26 \mathrm{a}$ & \\
\hline \multirow[t]{2}{*}{$\mathrm{CV}(\%)$} & \multicolumn{6}{|c|}{15,89} \\
\hline & \multicolumn{6}{|c|}{ Penicillium spp. (\%) } \\
\hline $2 \%$ & $18 \mathrm{Aa}$ & $13 \mathrm{Ab}$ & $21 \mathrm{Aa}$ & $4 \mathrm{Bc}$ & $10 \mathrm{Ab}$ & 13 \\
\hline $5 \%$ & $12 \mathrm{Ab}$ & $19 \mathrm{Aa}$ & $6 \mathrm{Bc}$ & $23 \mathrm{Aa}$ & $5 \mathrm{Bc}$ & 13 \\
\hline MD & 13 & 16 & 10 & 12 & 12 & \\
\hline \multirow[t]{2}{*}{$\mathrm{CV}(\%)$} & \multicolumn{6}{|c|}{17,92} \\
\hline & \multicolumn{6}{|c|}{ Sclerotinia spp. (\%) } \\
\hline $2 \%$ & $14^{\mathrm{ns}}$ & 39 & 30 & 32 & 6 & $24 \mathrm{~A}$ \\
\hline $5 \%$ & 24 & 26 & 28 & 17 & 20 & $23 \mathrm{~A}$ \\
\hline MD & $16 \mathrm{~b}$ & $32 \mathrm{a}$ & $25 \mathrm{a}$ & $18 \mathrm{~b}$ & $21 \mathrm{a}$ & \\
\hline $\mathrm{CV}(\%)$ & \multicolumn{6}{|c|}{15,27} \\
\hline
\end{tabular}

*interação significativa $\mathrm{e}^{\mathrm{ns}}$ interação não significativa dos fatores. Teste de médias não seguidas pela letra, maiúsculas na coluna e minúsculas na linha, diferem pelo teste de Tukey $(\mathrm{p}<0,05)$. Coeficiente de variação (CV), Média (MD).
Tabela 4. Índice de incremento de germinação (II.GER) e de emergência no campo (II.ECP) e índice de controle de sementes infestadas totais (IC.SIT) de cártamo submetidas a assepsia por diferentes a diferentes soluções sanitizantes de hipoclorito de sódio $(\mathrm{NaClO})$ e períodos de imersão.

\begin{tabular}{|c|c|c|c|c|c|}
\hline \multirow{3}{*}{$\mathrm{NaClO}$} & \multicolumn{5}{|c|}{ Períodos de imersão (min.) } \\
\hline & 1 & 5 & 10 & 15 & 30 \\
\hline & \multicolumn{5}{|c|}{ II.GER (\%) } \\
\hline $2 \%$ & 7,1 Bb* & $4,8 \mathrm{Bc}$ & $5,8 \mathrm{Bc}$ & $9,6 \mathrm{Aa}$ & 7,9 Bb \\
\hline $5 \%$ & $10,3 \mathrm{Ab}$ & $13,3 \mathrm{Aa}$ & 12,9 Aa & $9,6 \mathrm{Ab}$ & $9,3 \mathrm{Ab}$ \\
\hline \multirow[t]{2}{*}{$\mathrm{CV}(\%)$} & \multicolumn{5}{|c|}{3,19} \\
\hline & \multicolumn{5}{|c|}{ II.ECP (\%) } \\
\hline $2 \%$ & $15,0 \mathrm{Bb}^{*}$ & $13,4 \mathrm{Bc}$ & $16,2 \mathrm{Bb}$ & $21,8 \mathrm{Aa}$ & $16,5 \mathrm{Bb}$ \\
\hline $5 \%$ & $20,3 \mathrm{Aa}$ & $17,2 \mathrm{Ab}$ & $19,1 \mathrm{Aa}$ & $13,5 \mathrm{Bc}$ & $18,8 \mathrm{Ab}$ \\
\hline \multirow[t]{2}{*}{$\mathrm{CV}(\%)$} & \multicolumn{5}{|c|}{4,21} \\
\hline & \multicolumn{5}{|c|}{ IC.SIT (\%) } \\
\hline $2 \%$ & $8,2 \mathrm{Bc}^{*}$ & $55,1 \mathrm{Ba}$ & $40,0 \mathrm{Ab}$ & $48,4 \mathrm{Aa}$ & $43,3 \mathrm{Bb}$ \\
\hline $5 \%$ & $15,0 \mathrm{Ac}$ & $59,3 \mathrm{~A} \mathrm{a}$ & $37,1 \mathrm{Bb}$ & $41,9 \mathrm{Bb}$ & $60,0 \mathrm{Aa}$ \\
\hline $\mathrm{CV}(\%)$ & \multicolumn{5}{|c|}{9,01} \\
\hline
\end{tabular}

*interação significativa dos fatores. Teste de médias não seguidas pela letra, maiúsculas na coluna e minúsculas na linha, diferem pelo teste de Tukey $(\mathrm{p}<0,05)$. CV: coeficiente de variação. 